\title{
Effect of Correlated Temperature Fluctuations on the Phase Dynamics in an Ultrathin Lubricant Film
}

\author{
A. V. Khomenko \\ Sumy State University, Sumy, 40007 Ukraine \\ e-mail:khom@phe.sumdu.edu.ua \\ Received January 18, 2006; in final form, June 19, 2006
}

\begin{abstract}
The melting of an ultrathin lubricant film at friction between atomically smooth surfaces is studied with allowance for fluctuations of its temperature, which are described by the Ornstein-Uhlenbeck process. The behavior of the most probable types of shear stresses arising in the lubricant is considered, and phase diagrams for second- and first-order phase transformations (the melting of an amorphous lubricant and that of a crystalline lubricant, respectively) are constructed. It is shown that, in the former case, lubricant temperature fluctuations lead to the formation of a stick-slip friction domain separating the domains of dry and sliding friction, which is typical of first-order transitions. In the latter case, three domains of stick-slip friction arise, which mark the transitions between dry friction and metastable and stable sliding friction. As the time of correlation of lubricant temperature fluctuations gets longer, the temperature of rubbing surfaces rises to the point where sliding friction sets in.
\end{abstract}

PACS numbers: 68.35.Gy, 68.35.Rh

DOI: $10.1134 / \mathrm{S} 1063784207030061$

\section{INTRODUCTION}

Since smooth solid surfaces with a thin lubricant film in between are widely used in various fields of technology, sliding friction remains a subject of extensive research [1]. In this work, the author describes a transition mode of friction, which is observed in the case of an ultrathin (less than four molecular diameters thick) lubricant film between smooth or rough surfaces, high loads, and low shear rates. Experiments with mica, quartz, or metal oxide surfaces and also with monomolecular layers of surfactants with organic liquids or water solutions in between have shown that slide causes transformations of one dynamic phases into others $[2,3]$. These transformations show up as intermittent (stick-slip) friction [4-6], which is characterized by occasional transitions between two or more dynamic states in the course of steady slide and is the major reason for wear and failure of rubbing parts. Thus, molecularly thin lubricant films experience one or more types of transition, which gives rise to different modes of intermittent motion.

The reason for such anomalies in boundary friction has been elucidated, in particular, by experimentally studying the rheological properties of the lubricant film $[2,3]$. In those and similar studies, the lubricant is considered as an elastoviscous medium with a nonzero thermal conductivity. In [6], the behavior of the ultrathin lubricant film observed in experiments is treated in terms of the Landau-Ginzburg equation, in which the order parameter governs shear melting and solidification and a dynamic phase diagram showing the domains of sliding, stick-slip, and dry friction in the film temperature-film thickness coordinates was constructed.

In papers [7-9] extending work [6], an approach was developed according to which the transition of the ultrathin lubricant film from the solid-like to liquid-like phase is a result of thermodynamic and shear melting. A detailed analytical description of these processes was given under the assumption that they result from the self-organization of shear stress and strain fields, as well as of the lubricant temperature. The notion of additive noise of these quantities was introduced; phase diagrams where fluctuation intensities and the temperature of rubbing surfaces specify the domains of sliding, stick-slip, and dry friction were constructed; and the conditions under which the different friction modes are established in the system under the self-organized criticality regime were found.

However, while the lubricant condition is apparently controlled, as a rule, by the temperature, the issue of how correlated temperature fluctuations influence the friction process remains open. In this work, the author, invoking the Lorentz model of elastoviscous medium [7-9], shows that internal temperature fluctuations, which are described by the Ornstein-Uhlenbeck process, complicate the dynamic phase diagram. The steady-state regime of such a system is considered both in the case of continuous melting (melting of the amorphous lubricant; Section 3) and with regard to the 
deformation defect of the shear modulus (melting of the crystalline lubricant; Section 4). Also, the effect of the rubbing surface temperature on shear stresses arising between rubbing (moving) surfaces is considered, since these stresses are responsible for the phase state of the lubricant. As a result, different modes of stick-slip friction are predicted to occur in the system. The effect of fluctuation correlation time on the phase diagram is discussed.

\section{BASIC EQUATIONS}

In [7], the authors treated an elastoviscous medium with a nonzero thermal conductivity in rheological terms and derived a set of kinetic equations describing the correlated behavior of shear stresses $\sigma$, strain $\varepsilon$, and temperature $T$ in an ultrathin lubricant film during friction between atomically smooth mica surfaces. The basic assumption of he approach used in [7] is that the relaxation equation for $\sigma$ has the form

$$
\tau_{\sigma} \dot{\sigma}=-\sigma+G_{\varepsilon} .
$$

Here, the first term on the right stands for Debye relaxation for time $\tau_{\sigma} \equiv \eta_{\sigma} / G$, where $\eta_{\sigma}$ is the effective viscosity, $\left.G \equiv G(\omega)\right|_{\omega \rightarrow \infty}$ is the unrelaxed shear modulus, and $\omega$ is the circular frequency of a periodic external action. By substituting $\partial \varepsilon / \partial t$ for $\varepsilon / \tau_{\sigma}$, kinetic equation (1) reduces to a Maxwell-type expression for an elastoviscous medium, which is widely used in the theory of boundary friction [1]. In the stationary case, $\dot{\sigma}=0$, Eq. (1) passes into Hooke's law, $\sigma=G \varepsilon$.

The relaxation behavior of an elastoviscous lubricant during friction is also described by the KelvinVoigt equation [10]

$$
\dot{\varepsilon}=-\varepsilon / \tau_{\varepsilon}+\sigma / \eta,
$$

where $\tau_{\varepsilon}$ is the strain relaxation time and $\eta$ is the shear viscosity. The second term on the right describes the flow of a viscous fluid under the action of shear stresses. In the stationary case, $\dot{\varepsilon}=0$, we arrive at an expression similar to Hooke's law, $\sigma=G_{\varepsilon} \varepsilon$, where $G_{\varepsilon} \equiv$ $\eta /\left.\tau_{\varepsilon} \equiv G(\omega)\right|_{\omega \rightarrow 0}$ is the relaxed value of the shear modulus. Since formally Eq. (1) does not reduce to KelvinVoigt equation (2) $[10,11]$, it is assumed here that effective viscosity $\eta_{\sigma} \equiv \tau_{\sigma} G$ differs from real viscosity $\eta$. In addition, we will use simple approximations for the respective quantities: $G_{\varepsilon}(T), G(T), \eta_{\sigma}(T)=$ const and $\eta=\eta_{0}\left(T / T_{\mathrm{c}}-1\right)^{-1}$, where $\eta_{0} \equiv \eta\left(T=2 T_{\mathrm{c}}\right)$ is the characteristic value of the viscosity. The reason for such approximations is that $G_{\varepsilon}, G$, and $\eta_{\sigma}$ depend on temperature insignificantly and real $\eta$ diverges as the temperature decreases to its critical value $T_{\mathrm{c}}$ [12]. Taken together, Eqs. (1) and (2) represent a new rheological model. Note that the rheological properties of lubricant films are studied experimentally, which allows construction of phase diagrams (see, e.g., $[2,3]$ ).
According to the synergetic concept $[13,14]$, the set of Eqs. (1) and (2), which contain order parameter $\sigma$, conjugated field $\varepsilon$, and control parameter $T$, should be complemented by a kinetic equation for temperature. Using the basic relationships of the elasticity theory [7, $11]$, one can derive a heat conduction equation in the form

$$
\rho c_{\mathrm{V}} \dot{T}=\frac{\kappa}{l^{2}}\left(T_{\mathrm{s}}-T\right)+\sigma \dot{\varepsilon} .
$$

Here, $\rho$ is the lubricant density; $c_{\mathrm{V}}$ is the specific heat at constant volume; $\kappa$ is the thermal conductivity; $l$ is the heat conduction scale; $T_{\mathrm{s}}$ is the temperature of atomically smooth mica surfaces; and $\sigma=\sigma_{\mathrm{el}}+\sigma_{\mathrm{V}}$ is the total stress, which is the sum of the elastic, $\sigma_{\mathrm{el}}$, and viscous, $\sigma_{v}$, components. The first term on the right of (3) describes heat transfer from the lubricant film o the rubbing surface. The second one takes into account the dissipative heating of the stress-induced viscous flow and a heat source due to the reversible mechanocaloric effect, for which $T\left(\partial \sigma_{\mathrm{el}} / \partial T\right) \dot{\varepsilon} \equiv \sigma_{\mathrm{el}} \dot{\varepsilon}$ in the linear approximation.

Let $\sigma_{\mathrm{s}}=\left(\rho c_{\mathrm{V}} \eta_{0} T_{\mathrm{c}} / \tau_{T}\right)^{1 / 2}, \quad \varepsilon_{\mathrm{s}}=\sigma_{\mathrm{s}} / G_{0} \equiv$ $\left(\tau_{\varepsilon} / \tau_{T}\right)^{1 / 2}\left(\rho c_{\mathrm{V}} T_{\mathrm{c}} \tau_{\varepsilon} / \eta_{0}\right)^{1 / 2}$, and $T_{\mathrm{c}}$ be the units of measure for variables $\sigma, \varepsilon$, and $T$, respectively (here, $G_{0}=\eta_{0} / \tau_{\varepsilon}$, $\tau_{T} \equiv \rho l^{2} c_{\mathrm{V}} / \kappa$ is the time of heat conduction). Then, upon substitution of derivative $\dot{\varepsilon}$ (see (2)) into (3), basic equations (1)-(3) take the form

$$
\begin{gathered}
\tau_{\sigma} \dot{\sigma}=-\sigma+g \varepsilon, \\
\tau_{\varepsilon} \dot{\varepsilon}=-\varepsilon+(T-1) \sigma, \\
\tau_{T} \dot{T}=\left(T_{\mathrm{s}}-T\right)-\sigma \varepsilon+\sigma^{2}+\lambda(t),
\end{gathered}
$$

where constant $g=G / G_{0}$. Equations (4)-(6) are formally coincident with the Lorentz synergetic system $[13,14]$, which is used for description of both thermodynamic and kinetic transformations. In this work, the author takes into consideration the effect of temperature fluctuations on the lubricant evolution by introducing stochastic source $\lambda(t)$ into Eq. (6). This source represents the Ornstein-Uhlenbeck process [15],

$$
\langle\lambda(t)\rangle=0, \quad\left\langle\lambda(t) \lambda\left(t^{\prime}\right)\right\rangle=\frac{I}{\tau_{\lambda}} \exp \left(-\frac{\left|t-t^{\prime}\right|}{\tau_{\lambda}}\right),
$$

where $I$ is the fluctuation intensity and $\tau_{\lambda}$ is the time of fluctuation correlation.

Following [16], let us clarify the physical meaning of quantity $I$. The time correlation of the lubricant dimensionless temperature depends on the average value of product $\varphi(\tau)=\langle\Delta T(t) \Delta T(t+\tau)\rangle$, where $\Delta T(t)$ is the difference between the current and mean temperatures. Under the assumption that fluctuations $\Delta T$ are quasi-stationary, function $\varphi(\tau)$ takes the form

$$
\varphi(\tau)=\left\langle(\Delta T)^{2}\right\rangle \exp (-\zeta|\tau|)
$$


where $1 / \zeta$ is the relaxation time to equilibrium. Supposing that the time instants appearing in the above formulas are related as $t+\tau=t^{\prime}$ and that the mean square of temperature fluctuations is $\left\langle(\Delta T)^{2}\right\rangle=T^{2} / c_{\mathrm{V}}$, we get $\left\langle\Delta T(t) \Delta T\left(t^{\prime}\right)\right\rangle=\left(T^{2} / c_{\mathrm{V}}\right) \exp \left(-\zeta\left|t^{\prime}-t\right|\right)$. Comparing this expression with the second equality in (7) yields

$$
\lambda(t)=\Delta T(t), \quad \tau_{\lambda}=1 / \zeta, \quad I=T^{2} / c_{\mathrm{V}} \zeta .
$$

Thus, noise intensity $I$ depends on the temperature and specific heat $c_{\mathrm{V}}$ of the lubricant. At first glance, it appears that there exists a one-to-one correspondence between $I$ and $T_{\mathrm{s}}$ in the phase diagrams $T_{\mathrm{s}}(I)$ shown below and so a trajectory, instead of domains, must exist in the given coordinates, the motion along which describes the evolution of the system. However, such is not the case, since $T_{\mathrm{s}}$ is the temperature of a thermostat, which, varying arbitrarily, does not uniquely specify temperature $T$. One can only suppose that, due to the self-organization of the system, a certain value of $T_{\mathrm{s}}$ will be assigned different values of $T$ and, according to (8), different values of intensity $I$ at different time instants. In addition, $I$ varies as a result of time variation of $c_{\mathrm{V}}$. Thus, it is appropriate to speak of the existence of the phase diagram. Intensity $I$ can also be varied by varying parameter $\zeta$, which characterizes a specific system.

However, in this paper, temperature fluctuations are meant in the wider sense. The fact is that thermal actions are, as a rule, exerted by external stochastic sources. In addition, noise can equally be treated in terms of fluctuations of $T$ or $T_{\mathrm{s}}$. Such an interpretation is often used in simulation of noise in real physical systems [15].

In [7], melting of an ultrathin lubricant film between atomically smooth rubbing surfaces is viewed as a result of spontaneously arising shear stresses causing plastic flow when the rubbing surface is heated above critical value $T_{\mathrm{c} 0}=1+g^{-1}$. The initial reason for selforganization is positive feedback between $T$ (and $\sigma$ ) and $\varepsilon$ [5]. This feedback is due to the temperature dependence of the shear viscosity causing its divergence. On the other hand, the negative feedback between $\sigma$ (and $\varepsilon$ ) and $T$ in (6) is of great importance, since it makes the system stable.

According to such an approach, the lubricant represents a high-viscous medium that behaves as an amorphous solid, i.e., has a high effective viscosity and is still characterized by a yield stress $[2,3,11]$. The solidlike state of the lubricant corresponds to zero shear stresses $(\sigma=0)$, since Eq. (4) describing the elastic properties in the stationary case $(\dot{\sigma}=0)$ may be omitted in this case. Equation (5), which contains viscous stresses, reduces to the Debye law, which represents the relaxation of the shear strain for time $\tau_{\varepsilon}$. Finally, at $\lambda(t)=0$, heat conduction equation (6) takes the form of the simplest expression for temperature relaxation, which is free of terms describing dissipative heating and the mechanocaloric effect of the viscous fluid.

In the case of nonzero stresses $\sigma$, Eqs. (4)-(6) describe the properties inherent in the liquid-like state of the lubricant. Moreover, in the absence of shear strains, the rms displacement of molecules (atoms), according to [6], is given by $\left\langle u^{2}\right\rangle=T / G a$, where $a$ is the lattice constant. The rms displacement due to shear is found from the expression $\left\langle u^{2}\right\rangle=\sigma^{2} a^{2} / G^{2}$. The total $\mathrm{rms}$ displacement is the sum of these expressions provided that temperature fluctuations and stresses are independent from each other. This means that the lubricant melts as a result of heating and under the action of stresses generated by solid rubbing surfaces. Such a view is consistent with the consideration of solid-like state instability in terms of the theory of dynamic shear melting in the absence of temperature fluctuations [6]. It was shown [17] that the plastic flow of a lubricant layer takes place when it contains elastic stresses. In this case, shear stresses decrease the shear modulus of the lubricant. Consequently, the friction force decreases with increasing relative velocity $V=l \partial \varepsilon / \partial t$ of rubbing surfaces, since friction causes shear stresses to grow, according to the Maxwell-type stress-strain relation$\operatorname{ship} \partial \sigma / \partial t=-\sigma / \tau_{\sigma}+G \partial \varepsilon / \partial t$. We will assume that, as the temperature rises, the lubricant film becomes more liquid-like and the friction force declines because of a decrease in the molecular hop activation energy.

In this work, the author studies the effect of stochastic source $\lambda(t)$ on the evolution of stress $\sigma(t)$. According to experimental data for organic lubricants $[2,3,6]$, the stress relaxation time under normal pressure is $t_{\sigma} \sim$ $10^{-10} \mathrm{~s}$ and grows with pressure by several orders of magnitude. Since the ultrathin lubricant film is less than four molecular layers thick, the temperature relaxes to $T_{\mathrm{s}}$ for a time satisfying the inequality $\tau_{T} \ll \tau_{\sigma}$ [7]. Therefore, we assume that the adiabatic conditions

$$
\tau_{\sigma} \approx \tau_{\varepsilon} \gg \tau_{T}
$$

under which lubricant temperature $T$ varies with the shear components of stress $\sigma$ and strain $\varepsilon$, are met. Then, one can separate out a small parameter in Eq. (6) and put $\tau_{T} \dot{T}=0$. Eventually, we come to an expression for the temperature,

$$
T=T_{\mathrm{s}}-\sigma \varepsilon+\sigma^{2}+\lambda(t) .
$$

Let us simplify the set of Eqs. (4), (5), and (10) by reducing it for a single equation in shear strength $\sigma(t)$. To this end, $\varepsilon$ and $T$ should be expressed through $\sigma$. Differentiating an expression in strain $\varepsilon$ derived from (4) with respect of time, we obtain an expression in $\dot{\varepsilon}$. Substituting the expression for $\varepsilon$, the resulting expression for $\dot{\varepsilon}$, and (10) into (5) yields an evolutionary equation in the form of the canonic nonlinear equation for a stochastic oscillator of the van der Pol oscillator type,

$$
m \ddot{\sigma}+\gamma(\sigma) \dot{\sigma}=f(\sigma)+\phi(\sigma) \lambda(t),
$$


where parameter $m$, friction coefficient $\gamma$, force $f$, and noise amplitude $\phi$ are given by

$$
\begin{gathered}
m \equiv \frac{\tau_{\sigma} \tau_{\varepsilon}}{g}, \gamma(\sigma) \equiv \frac{1}{g}\left[\tau_{\varepsilon}+\tau_{\sigma}\left(1+\sigma^{2}\right)\right], \\
f(\sigma) \equiv \sigma\left(T_{\mathrm{s}}-1-g^{-1}\right)-\sigma^{3}\left(g^{-1}-1\right), \phi(\sigma) \equiv \sigma .
\end{gathered}
$$

\section{EFFECTIVE POTENTIAL METHOD}

Let us find the distribution of the system with time $t$ in the phase space that is parametrized by generalized coordinate $\sigma$ and momentum $p \equiv m \dot{\sigma}$. To this end, we will make use of the effective potential method that has been developed in [18-20]. Euler equation (11) can be represented in the Hamiltonian form as follows:

$$
\begin{gathered}
\dot{\sigma}=m^{-1} p, \\
\dot{p}=-m^{-1} \gamma(\sigma) p+f(\sigma)+\phi(\sigma) \lambda(t) .
\end{gathered}
$$

Statistical investigation implies determination of function $\Pi(\sigma, p, t)$, which is the probability density of the presence of stress $\sigma$ and its derivative $\dot{\sigma}$ at given time instant $t$. In other words, it is $\lambda$-averaged ( $\lambda$ is the noise intensity) distribution function $\rho(\sigma, p, t)$ of solutions to system (13),

$$
\Pi(\sigma, p, t)=\langle\rho(\sigma, p, t)\rangle .
$$

We assume that function $\rho=\rho(\sigma, p, t)$ satisfies the continuity equation

$$
\frac{\partial \rho}{\partial t}+\left[\frac{\partial}{\partial \sigma}(\dot{\sigma} \rho)+\frac{\partial}{\partial p}(\dot{p} \rho)\right]=0 .
$$

Substituting equalities (13) into (15), we come to the Liouville equation

$$
\frac{\partial \rho}{\partial t}=(\hat{L}+\hat{N} \lambda) \rho
$$

with operators

$$
\hat{L} \equiv-\frac{p}{m} \frac{\partial}{\partial \sigma}-\frac{\partial}{\partial p}\left(f-\frac{\gamma}{m} p\right), \quad \hat{N} \equiv-\phi \frac{\partial}{\partial p} .
$$

In terms of the interaction representation, a microscopic distribution function is given by $\bar{\sigma}=e^{-\hat{L} t} \rho$, so that Eq. (16) takes the form $\partial \varpi / \partial t=\hat{\mathfrak{R}} \Phi$, where $\hat{\mathfrak{R}} \equiv$ $\lambda\left(e^{-\hat{L} t} \hat{N} e^{\hat{L} t}\right)$. Use of the accumulant expansion method [21] leads to the linear differential kinetic equation $\partial\langle\varpi\rangle(t) / \partial t=\left[\int_{0}^{t}\left\langle\hat{\mathfrak{R}}(t) \hat{\mathfrak{R}}\left(t^{\prime}\right)\right\rangle d t^{\prime}\right]\langle\varpi\rangle(t)$ up to terms $O\left(\hat{\mathfrak{R}}^{2}\right)$. The transition from the interaction representa- tion to the starting one yields for distribution function (14)

$$
\begin{gathered}
\frac{\partial}{\partial t} \Pi(t) \\
=\left\{\hat{L}+\int_{0}^{t}\langle\lambda(\tau) \lambda(0)\rangle\left[\hat{N}\left(e^{\hat{L} \tau} \hat{N} e^{-\hat{L} \tau}\right)\right] d \tau\right\} \Pi(t) .
\end{gathered}
$$

Since physical time $t$ far exceeds noise correlation time $\tau_{\lambda}$, one can set the upper limit of integration equal to infinity. Then, expanding the exponentials in (18), we get the expression

$$
\frac{\partial \Pi}{\partial t}=(\hat{L}+\hat{\Lambda}) \Pi
$$

where dissipative operator

$$
\hat{\Lambda} \equiv \sum_{n=0}^{\infty} \hat{\Lambda}^{(n)}, \quad \hat{\Lambda}^{(n)} \equiv C^{(n)}\left(\hat{N} \hat{L}^{(n)}\right)
$$

is determined by commutators according to the recurrence formula

$$
\hat{L}^{(n+1)}=\left[\hat{L}, \hat{L}^{(n)}\right], \quad \hat{L}^{(0)} \equiv \hat{N}
$$

$([\hat{A}, \hat{B}]=\hat{A} \hat{B}-\hat{B} \hat{A})$ and by the moments

$$
C^{(n)}=\frac{1}{n !} \int_{0}^{\infty} \tau^{n}\langle\lambda(\tau) \lambda(0)\rangle d \tau
$$

of correlation function (7). The first moments are

$$
C^{(0)}=I, \quad C^{(1)}=I \tau_{\lambda} .
$$

In the general case, further consideration is impossible; therefore, we make the simplifying assumption, i.e., separate out small parameter $\epsilon \ll 1$, which coincides with the Kubo number [21]. Putting $m=\epsilon^{2}$ in Eq. (11) and measuring the generalized momentum in units of $\epsilon$, we will analyze the case of an overdamped oscillator in which the sliding friction force exceeds the other components by $\epsilon^{-1} \gg 1$ times. Then, Eqs. (13) take the form

$$
\begin{aligned}
\frac{\partial \sigma}{\partial t} & =\epsilon^{-1} p, \quad \frac{\partial p}{\partial t}=-\epsilon^{-2} \gamma(\sigma) p \\
& +\epsilon^{-1}[f(\sigma)+\phi(\sigma) \lambda(t)] .
\end{aligned}
$$

Accordingly, the Fokker-Planck equation (19) can be written as

$$
\left(\frac{\partial}{\partial t}-\hat{L}\right) \Pi=\epsilon^{-2} \hat{\Lambda} \Pi,
$$

where operator $\hat{L} \equiv \epsilon^{-1} \hat{L}_{1}{ }^{+} \epsilon^{-2} \hat{L}_{2}$ has two components: $\hat{L}_{1} \equiv-p(\partial / \partial \sigma)-f(\partial / \partial p)$ and $\hat{L}_{2} \equiv \gamma(\partial / \partial p) p$. Dissipative operator $\hat{\Lambda}$ is given by (17) and (20)-(22) and, up to 


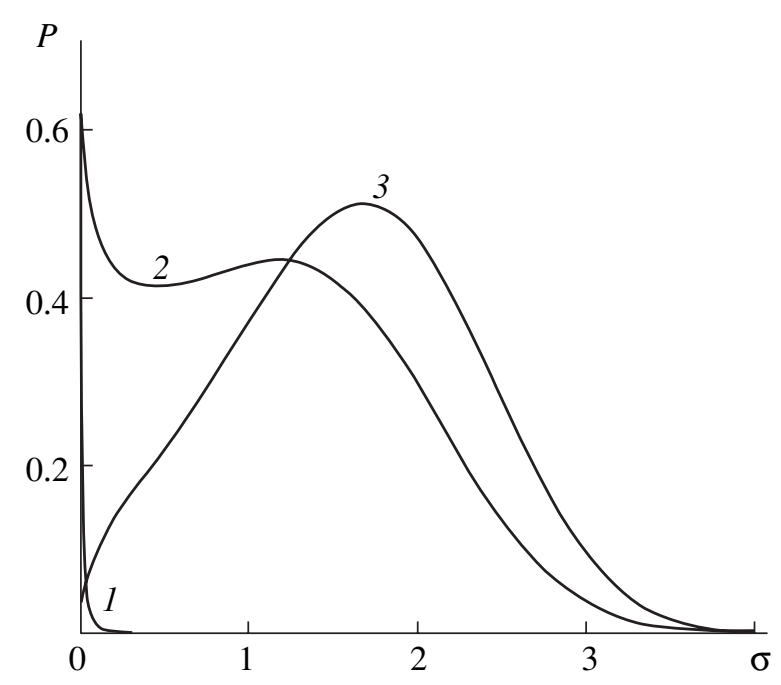

Fig. 1. Shear stress distribution function for the secondorder transition at $g=0.2, \tau_{\sigma}=\tau_{\varepsilon}=0.1 ; \tau_{\lambda}=0.2 ; l=5$; and $T_{\mathrm{S}}=(1) 5$, , (2) 16, and (3) 20 .

terms of the second order of smallness, has the form

$$
\begin{gathered}
\hat{\Lambda}=\left(C^{(0)}+\gamma C^{(1)}\right) \phi^{2} \frac{\partial^{2}}{\partial p^{2}} \\
+\epsilon C^{(1)} \phi^{2}\left[-\frac{1}{\phi}\left(\frac{\partial \phi}{\partial \sigma}\right)\left(\frac{\partial}{\partial p}+p \frac{\partial^{2}}{\partial p^{2}}\right)+\frac{\partial^{2}}{\partial \sigma \partial p}\right]+O\left(\epsilon^{2}\right) .
\end{gathered}
$$

Since melting of the lubricant is characterized by stress $\sigma$ and time $t$, we will consider the projection of the distribution function on the half-space $(\sigma, t)$. To this end, we pass to the Fokker-Planck equation in function $P(\sigma, t)$, using the moments of the initial distribution $P_{n}(\sigma, t) \equiv \int p^{n} \Pi(\sigma, p, t) d p$, the zeroth of which gives the desired result. Multiplying Eq. (25) by $p^{n}$ and integrating the result over all momenta, we arrive at the recurrence relation

$$
\begin{gathered}
\epsilon^{2} \frac{\partial P_{n}}{\partial t}-n \gamma P_{n}+\epsilon\left(\frac{\partial P_{n+1}}{\partial \sigma}+n f P_{n-1}\right) \\
=n(n-1)\left(C^{(0)}+\gamma C^{(1)}\right) \phi^{2} P_{n-2} \\
+\epsilon n C^{(1)}\left[\phi^{2} \frac{\partial P_{n-1}}{\partial \sigma}-n \phi\left(\frac{\partial \phi}{\partial \sigma}\right) P_{n-1}\right]+O\left(\epsilon^{2}\right) .
\end{gathered}
$$

Next, applying the hierarchical approach to (27), we write the Fokker-Planck equation. For $n=0$, we get an equation for desired function $P=P_{0}(\sigma, t)$,

$$
\frac{\partial P}{\partial t}=-\epsilon^{-1} \frac{\partial P_{1}}{\partial \sigma}
$$

First-order moment $P_{1}$ is given by the expression

$$
P_{1}=\frac{\epsilon}{\gamma}\left\{f P+\frac{\partial P_{2}}{\partial \sigma}-C^{(1)}\left[\phi^{2} \frac{\partial P}{\partial \sigma}-\phi\left(\frac{\partial \phi}{\partial \sigma}\right) P\right]\right\},
$$

which follows from (27) where $n=1$ and terms of the first order of smallness in $\epsilon \ll 1$ are included. Taking into account terms of the zeroth order in $\epsilon$ at $n=2$ gives an expression for the second-order moment $P_{2}$,

$$
P_{2}=-\left(\frac{C^{(0)}}{\gamma}+C^{(1)}\right) \phi^{2} P
$$
form

As a result, the Fokker-Planck equation takes the

$$
\frac{\partial P}{\partial t}=-\frac{\partial}{\partial \sigma}\left(D^{(1)} P\right)+\frac{\partial}{\partial \sigma}\left(D^{(2)} \frac{\partial P}{\partial \sigma}\right)
$$

where

$$
\begin{gathered}
D^{(1)}=\frac{1}{\gamma}\left\{f-C^{(0)} \phi^{2} \frac{\partial \gamma^{-1}}{\partial \sigma}-\phi \frac{\partial \phi}{\partial \sigma}\left(\frac{2 C^{(0)}}{\gamma}+C^{(1)}\right)\right\}, \\
D^{(2)}=\frac{\phi^{2}}{\gamma}\left(\frac{C^{(0)}}{\gamma}+2 C^{(1)}\right)
\end{gathered}
$$

are the drift and diffusion coefficients, respectively.

The stationary solution of Eq. (31) yields the distribution

$$
P(\sigma)=Z^{-1} \exp \left(\int_{0}^{\sigma} \frac{D^{(1)}(x)}{D^{(2)}(x)} d x\right),
$$

where $Z=\int_{0}^{\infty} d \sigma \exp \left\{\int_{0}^{\sigma}\left[D^{(1)}(x) / D^{(2)}(x)\right] d x\right\}$ is a normalizing factor.

\section{CONTINUOUS TRANSFORMATION}

Distribution (33) (Fig. 1) has maxima the positions of which depend on a relation between parameters $T_{\mathrm{s}}$, $g, I, \tau_{\lambda}, \tau_{\varepsilon}$, and $\tau_{\sigma}$. When the temperature of rubbing surfaces is small, a single peak is observed at $\sigma_{0}=0$, which corresponds to the solid-like state of the lubricant and dry friction (curve 1 ). As $T_{\mathrm{s}}$ grows, a maximum appears at some point $\sigma_{0} \neq 0$ meeting the steady state in which shear stresses spontaneously arise, causing the lubricant film to melt and, accordingly, the sliding friction regime to set in (curve 2). As $T_{\mathrm{s}}$ grows further, the maximum at $\sigma=0$ disappears (curve 3 ).

The coexistence of the maxima of $P(\sigma)$, which correspond to the zero and nonzero values of the stress, manifests the regime of stick-slip motion, i.e., the regime when dry friction from time to time changes to sliding friction and vice versa. Such behavior is typical of the melting of a lubricant comprising a mixture of the solid- and liquid-like phases. According to $[8,9]$, 
such a situation may happen even if temperature $T_{\mathrm{s}}$ of rubbing surfaces equals zero provided that the intensity of additive strain fluctuations exceeds critical value $I_{\varepsilon}=$ $\left(1+2 g^{-1}\right) / 2 g$. In this case, the behavior of the system is alike to that observed under the conditions of self-organized criticality, when self-organization may occur spontaneously (without external actions) [22], unlike in the case of the phase transition.

The state of shear stress is steady when distribution (33) reaches an extremum,

$$
\frac{D^{(1)}(\sigma)}{D^{(2)}(\sigma)}=0 .
$$

Substituting (12), (23), and (32) into (34) yields

$$
\begin{gathered}
{\left[T_{\mathrm{s}}-\frac{1+g}{g}-\sigma^{2}\left(\frac{1-g}{g}\right)-I \tau_{\lambda}\right]} \\
\times\left[\tau_{\varepsilon}+\tau_{\sigma}\left(1+\sigma^{2}\right)\right]^{2}-2 \operatorname{Ig}\left(\tau_{\varepsilon}+\tau_{\sigma}\right)=0 .
\end{gathered}
$$

A solution to (35) is shown in Fig. 2, which shows that an increase in noise intensity $I$ causes a two-valued region to appear in the monotonic curve $\sigma_{0}\left(T_{\mathrm{s}}\right)$, which is typical of first-order transitions. Putting $\sigma=0$ in (35), we find a critical value of the rubbing surface temperature,

$$
T_{\mathrm{s} 0}=\frac{1+g}{g}+\left(\tau_{\lambda}+\frac{2 g}{\tau_{\varepsilon}+\tau_{\sigma}}\right) I
$$

above which the system exhibits sliding friction. It is seen that $T_{\mathrm{s} 0}$ grows with noise intensity $I$ and correlation time $\tau_{\lambda}$. The stress and strain relaxation times influence $T_{\mathrm{e} 0}$ inversely. In the phase diagram shown in Fig. 3, one can separate the domains of dry friction (DF), sliding friction (SF), and stick-slip friction (SS). As correlation time $\tau_{\lambda}$ increases, so does the value of $T_{\mathrm{s}}$ meeting tricritical point $T$ at given noise intensity $I$. Clearly, the DF domain expands in this case, while the probability of sliding friction and stick-slip friction decreases.

\section{MAKING ALLOWANCE \\ FOR THE DEFORMATION DEFECT \\ OF THE SHEAR MODULUS}

Actually, the shear modulus of the lubricant appearing in Eq. (4) through relaxation time $\tau_{\sigma}$ is stressdependent [17]. Therefore, elastic deformation changes to plastic deformation when the shear stress reaches characteristic value $\sigma_{\mathrm{p}}$ not exceeding $\sigma_{\mathrm{s}}$ (otherwise, plastic flow does not occur). To allow for the deformation defect of the shear modulus, we will make use of the dependence $\tau_{\sigma}(\sigma)$ suggested in [7] instead of using stress-independent $\tau_{\sigma}$. Then, Eq. (4) takes the form

$$
\tau_{\mathrm{p}} \dot{\sigma}=-\sigma\left(1+\frac{\theta^{-1}-1}{1+\sigma / \alpha}\right)+g_{\Theta} \varepsilon
$$

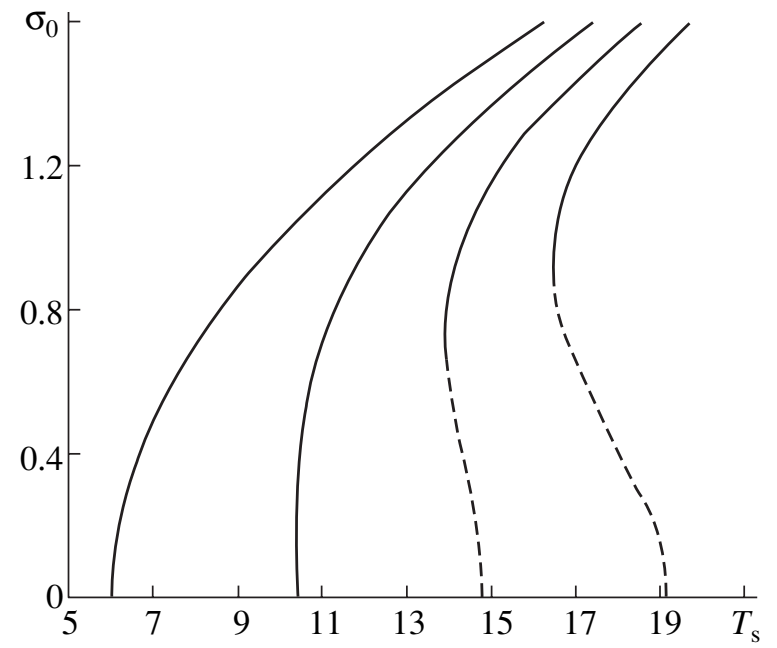

Fig. 2. Steady-state value of shear stress $\sigma_{0}$ vs. temperature $T_{\mathrm{S}}$ for the same parameters as in Fig. 1 and $I=0,2,4$, and 6 (for the curves from left to right).

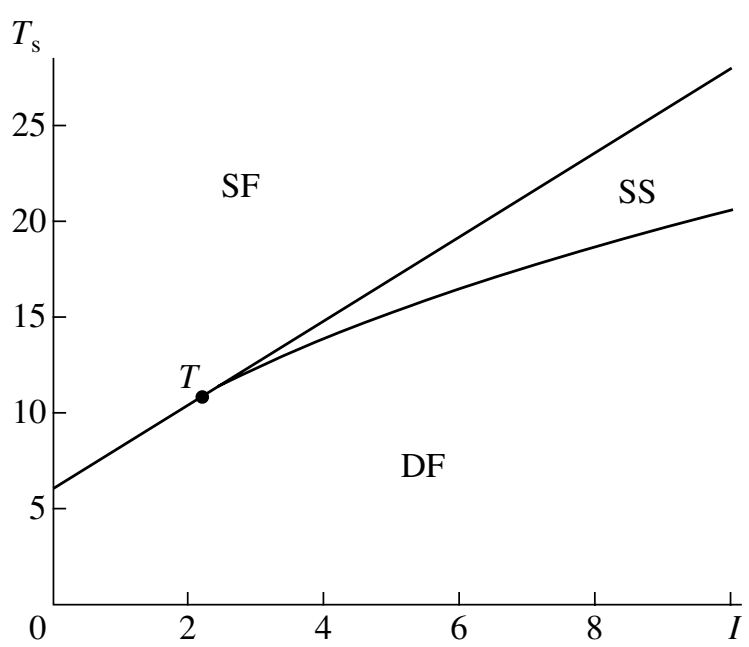

Fig. 3. Phase diagram for the parameters in Fig. 1. SF, sliding friction; DF, dry friction; and SS, stick-slip frictions ( $T$ is the tricritical point).

where $\tau_{p}=\eta_{\sigma} / \Theta$ is the plastic flow relaxation time, $\Theta$ is the hardening parameter, $\theta=\Theta / G<1$ is the parameter relating the slopes of the stress-strain curve in its plastic and Hooke's portions, and $g_{\Theta}=G^{2} / \Theta G_{0}$ and $\alpha=$ $\sigma_{\mathrm{p}} / \sigma_{\mathrm{s}}$ are constants. Then, with regard to approximation (9), the system of Eqs. (37), (5), and (6) reduces, as before, to Eq. (11), where parameter $m$, friction coefficient $\gamma$, force $f$, and noise amplitude $\phi$ are given by

$$
\begin{gathered}
m \equiv \frac{\tau_{\mathrm{p}} \tau_{\varepsilon}}{g_{\Theta}} \\
\gamma(\sigma) \equiv \frac{1}{g_{\Theta}}\left[\tau_{\varepsilon}\left(1+\frac{\theta^{-1}-1}{(1+\sigma / \alpha)^{2}}\right)+\tau_{\mathrm{p}}\left(1+\sigma^{2}\right)\right] \\
f(\sigma) \equiv \sigma\left[T_{\mathrm{s}}-1-\frac{1}{g_{\Theta}}\left(\frac{\theta^{-1}+\sigma / \alpha}{1+\sigma / \alpha}\right)\right]
\end{gathered}
$$



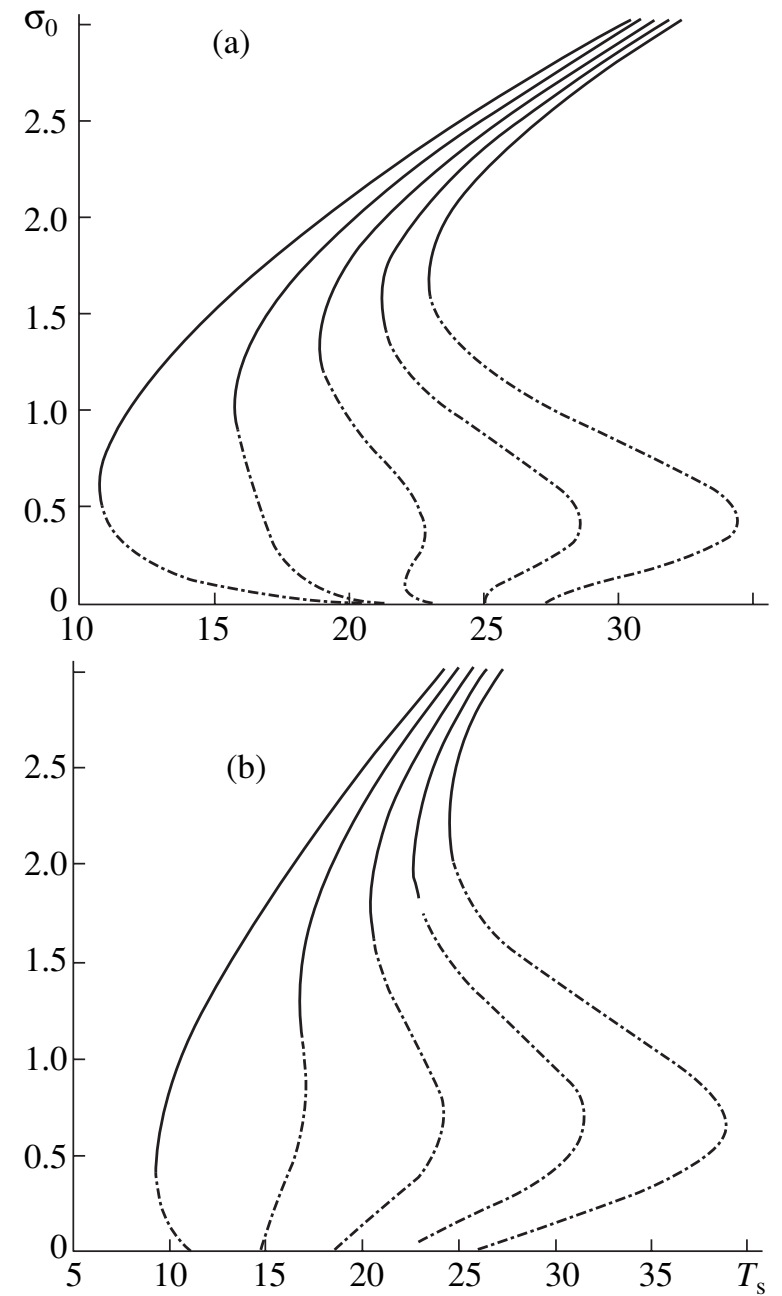

Fig. 4. Steady-state value of shear stress $\sigma_{0}$ vs. temperature $T_{\mathrm{s}}$ for the first-order transition at $\tau_{\mathrm{p}}=\tau_{\varepsilon}=\tau_{\lambda}=0.1, \theta^{-1}=7$, and $I=0,2,4,6$, and 8 (for the curves from left to right): (a) $g_{\Theta}=0.4, \alpha=0.3$ and (b) $g_{\Theta}=0.7, \alpha=0.8$.

$$
\begin{gathered}
-\sigma^{3}\left[\frac{1}{g_{\Theta}}\left(\frac{\theta^{-1}+\sigma / \alpha}{1+\sigma / \alpha}\right)-1\right], \\
\phi(\sigma) \equiv \sigma .
\end{gathered}
$$

In accordance with the approach stated in Section 2, condition (34), under which the state of shear stresses becomes steady, takes the form (in view of (23), (38), and (32))

$$
\begin{gathered}
\left\{T_{\mathrm{s}}-1-\frac{1}{g_{\Theta}}\left(\frac{\theta^{-1}+\sigma / \alpha}{1+\sigma / \alpha}\right)\right. \\
\left.-\sigma^{2}\left[\frac{1}{g_{\Theta}}\left(\frac{\theta^{-1}+\sigma / \alpha}{1+\sigma / \alpha}\right)-1\right]-I \tau_{\lambda}\right\} \gamma^{2}-2 I_{\gamma} \\
+\frac{2 I_{\sigma}}{g_{\Theta}}\left[\tau_{\mathrm{p}} \sigma-\tau_{\varepsilon} \frac{\theta^{-1}-1}{\alpha(1+\sigma / \alpha)^{3}}\right]=0 .
\end{gathered}
$$
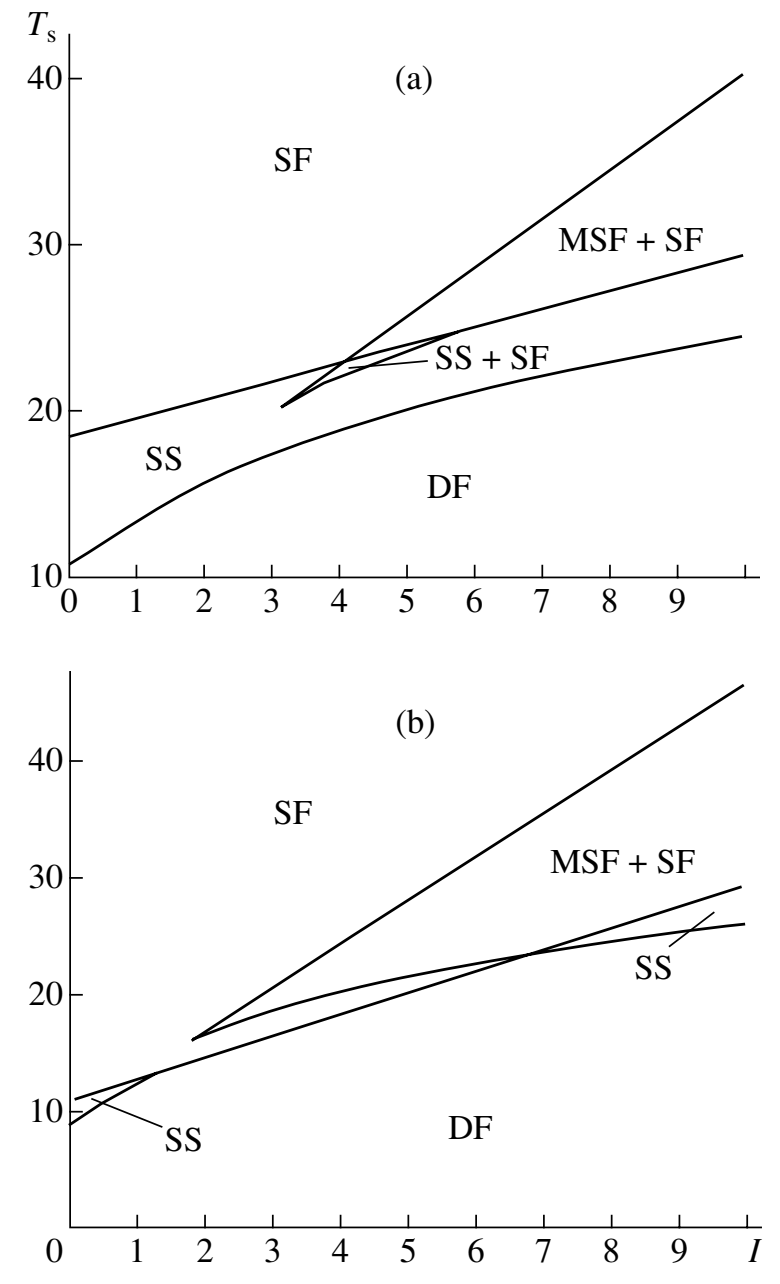

Fig. 5. Phase diagrams for the same parameters as in Fig. 4: SF, sliding friction; DF, dry friction; SS, stick-slip friction; and MSF, metastable sliding friction.

Putting $\sigma=0$ in (39), we find an equality (similar in sense to (36)) that specifies a limiting value of $T_{\mathrm{s}}$ at which the maximum in distribution (33) still exists at zero stresses corresponding to the solid-like state,

$$
T_{\mathrm{s} 0}=\frac{\theta^{-1}+g_{\Theta}}{g_{\Theta}}+\left(\tau_{\lambda}+\frac{2 g_{\Theta}}{\theta^{-1} \tau_{\varepsilon}+\tau_{\mathrm{p}}}\right) I .
$$

The dependences of the steady-state values of shear stresses on the rubbing surface temperature, $\sigma_{0}\left(T_{\mathrm{s}}\right)$, which are solutions to Eq. (39), are shown in Fig. 4. It is seen that an increase in noise intensity $I$ gives rise to two steady states, which correspond to the maxima of distribution function $P(\sigma)$ (see (33)) at nonzero values of $\sigma$. Thus, one can conclude that the lubricant melts at two steady-state values of the stress [7], which depend on $T_{\mathrm{s}}, g_{\Theta}, I, \theta, \alpha, \tau_{\lambda}, \tau_{\mathrm{p}}$, and $\tau_{\varepsilon}$. The smaller one meets the metastable liquid-like state of the lubricant (dashed curves); the larger (continuous curves), its stable liquidlike state. These state are separated by the unstable state 
(dash-and-dot curves), which is related to the minimum of probability (33).

The phase diagrams inherent to this system are depicted in Fig. 5. Here, the DF domain corresponds to only one maximum of probability $P(\sigma)$ at a zero value of stress $\sigma$. This maximum meets the solid-like state of the lubricant, or the dry friction conditions $[7,8]$. In the SS domain, the solid-like and liquid-like phases coexist; that is, one more peak (along with that at zero $\sigma$ ) arises in the $P(\sigma)$ curve, which meets the melting of the lubricant, or the sliding friction conditions. In the SS + SF domain, the run of the $P(\sigma)$ curve is most complicated. Here, the solid-like state of the lubricant coexists with the metastable and stable liquid-like states corresponding to maxima of $P(\sigma)$. Accordingly, the stickslip friction conditions may set in, under which these three dynamic regimes occasionally pass into each other. It should be noted that this domain, unlike the others, cannot be realized if the parameters of the system change (Fig. 5b). The SF domain corresponds to stable sliding friction, i.e., to the liquid-like phase of the lubricant at a single steady-state value of he stress. In the intermittent MSF + SF domain, the metastable and stable regimes of sliding friction occasionally change each other. Remarkably, when the transition from the $\mathrm{SS}+\mathrm{SF}$ domain to the MSF + SF domain takes place, dry friction completely disappears. As noise correlation time $\tau_{\lambda}$ increases, the DF domain expands and the SF domain shrinks.

\section{CONCLUSIONS}

Thus, it is shown that, as the temperature of rubbing surfaces grows, the system exhibits signs of self-organization, as a result of which the sliding friction conditions set in. Here, the degree of correlation of temperature variation over the lubricant is essential. If correlation time $\tau_{\lambda}$ grows at a given temperature fluctuation intensity, the rubbing surface temperature must increase for the dry-to-sliding friction transition to take place. In the case of continuous transformation at low intensities, this transition occurs without forming the stick-slip friction domain, i.e., proceeds as a secondorder transition (melting of the amorphous lubricant). When the fluctuation intensity is high, the first ordertransition (melting of the crystalline lubricant) is observed.

The first-order transition was described with allowance for the deformation defect of the shear modulus. It was shown that a change in the fluctuation intensity and/or in the rubbing surface intensity may transfer the system from the dry friction regime to the regime of sliding friction, with the latter arising at two values of the shear stress. The respective phase diagram exhibits the domains of stick-slip friction, where metastable sliding friction changes occasionally to stable sliding friction.

\section{ACKNOWLEDGMENTS}

The author thanks Ya.A. Lyashenko for fruitful discussion.

This work was supported by a grant of the Ministry of Ukraine.

\section{REFERENCE}

1. B. N. J. Persson, Sliding Friction: Physical Principles and Applications (Springer, Berlin, 1998).

2. H. Yoshizawa, Y.-L. Chen, and J. Israelachvili, J. Phys. Chem. 97, 4128 (1993).

3. H. Yoshizawa and J. Israelachvili, J. Phys. Chem. 97, 11300 (1993).

4. E. D. Smith, M. O. Robbins, and M. Cieplak, Phys. Rev. B 54, 8252 (1996).

5. J. Krim, D. H. Solina, and R. Chiarello, Phys. Rev. Lett. 66, 181 (1991).

6. I. S. Aranson, L. S. Tsimring, and V. M. Vinokur, Phys. Rev. B 65, 125402 (2002).

7. A. V. Khomenko and O. V. Yushchenko, Phys. Rev. E 68, 036110 (2003).

8. A. V. Khomenko, Phys. Lett. A 329, 140 (2004).

9. A. V. Khomenko and Ya. A. Lyashenko, Zh. Tekh. Fiz. 75 (11), 17 (2005) [Tech. Phys. 50, 1408 (2005)].

10. Rheology: Theory and Applications, F. R. Eirich, Ed. (Academic, New York, 1956; Inostrannaya Literatura, Moscow, 1962).

11. L. D. Landau and E. M. Lifshitz, Course of Theoretical Physics, Vol. 7: Theory of Elasticity (Nauka, Moscow, 1987; Pergamon, New York, 1986).

12. A. I. Olemskoŭ and E. A. Toropov, Fiz. Met. Metalloved., No. 9, 5 (1991).

13. H. Haken, Synergetics: An Introduction (Springer, Berlin, 1977; Mir, Moscow, 1980).

14. A. I. Olemskoı̆ and A. V. Khomenko, Zh. Éksp. Teor. Fiz. 110, 2144 (1996) [JETP 83, 1180 (1996)].

15. C. W. Gardiner, Handbook of Stochastic Methods (Springer, Berlin, 1983; Mir, Moscow, 1985).

16. L. D. Landau and E. M. Lifshitz, Course of Theoretical Physics, Vol. 5: Statistical Physics, 4th ed. (Nauka, Moscow, 1995; Butterworth, London, 1999), Chap. 1.

17. V. L. Popov, Zh. Tekh. Fiz. 71 (5), 100 (2001) [Tech. Phys. 46, 605 (2001)].

18. D. O. Kharchenko, Ukr. Phys. J. 44, 647 (1999).

19. A. V. Khomenko, D. O. Kharchenko, and O. V. Yushchenko, Vestn. Lvovsk. Univ., No. 37, 44 (2004).

20. O. V. Yushchenko, Candidate's Dissertation (Sumy State University, Sumy, 2004).

21. N. G. Van Kampen, Stochastic Processes in Physics and Chemistry (North-Holland, Amsterdam, 1981; Vysshaya Shkola, Moscow, 1990).

22. P. Bak, How Nature Works: the Science of Self-Organized Criticality (Springer, New York, 1996).

Translated by V. Isaakyan 